\title{
Investigation of the Heat Transfer Coefficient of Liquid and Gas Bubble Train Flow in a Square Mini-channel Using Infra-Red thermography
}

\author{
by Balkrishna Mehta, Sameer Khandekar* \\ * Department of Mechanical Engineering, Indian Institute of Technology Kanpur \\ Kanpur 208016 (UP), India \\ *samkhan@iitk.ac.in
}

\begin{abstract}
This paper experimentally investigates the heat transfer in mini-channel of square cross-section $(5 \mathrm{~mm} \times 5 \mathrm{~mm}$; aspect ratio $=1.0 ; D_{h}=5 \mathrm{~mm}, \mathrm{Bo} \approx 3.4$ ) in non-boiling horizontal Taylor bubble-train slug flow under the uniform heat flux condition using Infrared Thermography (IRT). Due to relatively high Bo, gravity force affects the thermo-hydrodynamics of the flow. The important governing parameters are lengths of liquid slug and bubbles, liquid and gas superficial velocities which depend on the volume flow ratio ' $\beta$ '. In this work, the liquid superficial velocity is kept constant to 0.054 $\mathrm{m} / \mathrm{s}$ and volume flow ratio varies from 0.4 to 0.7 .
\end{abstract}

\section{Introduction}

Taylor bubble flow is one of the sub-classes of conventional slug flow. Taylor slug flow conditions are typically characterized by a sequence of long bubbles which are trapped in between liquid slugs; such flows therefore have strong geometric constraints. This special pattern appears when surface tension dominates over gravitational body force, typically in mini/micro channels. In such geometries, the flow is essentially laminar and predominantly viscous. The shapes and sizes of the liquid slugs and bubbles are fundamentally governed in the resultant of the gravity, surface tension and viscous effect. Interplay between the gravity and the surface tension forces, mainly depends on the size of the channel $(\mathrm{Bo})$ for specific liquid and gas phase. At relatively high $\mathrm{Bo}\left(\mathrm{Bo}_{\mathrm{B}} \mathrm{B} \mathrm{c}_{\mathrm{cr}} \approx 1.835\right)$ systems gravity force dominates over the surface tension, the liquid film essentially takes the lower part of the channel cross-section whereas at the upper part, negligibly thin liquid film exists. The understanding of species transport under such a flow configuration is a challenging problem. In recent years, research on Taylor bubbles has increased due to the development of mini/micro scale systems in diverse branches of engineering wherein Taylor bubble flow is the dominant flow pattern, [1, 2, 3, 4, 5]. The definitive pioneering work on Taylor bubble flows, for configurations involving conventional circular channels is that of [6], who observed that a Taylor bubble does not rise spontaneously in a water filled vertical capillary under the effect of gravity for Bo < 1.835. [7] observed that when a gas bubble displaces a wetting fluid which is filled in a horizontal capillary, a liquid film is deposited on the capillary between the gas bubble and the inside wall. This film thickness follows the scaling $(\delta / R) \sim \mathrm{Ca}^{1 / 2}$ for Ca ranging from $10^{-5}$ to $10^{-1}$. Semi-analytical work, with some simplified assumptions by [6] established the scaling as $(\delta / \mathrm{R}) \sim \mathrm{Ca}^{2 / 3}$, the theory being valid for creeping flows at low Ca ranging from $10^{-3}$ to $10^{-2}$ and bubble Weber number, We $<<1$. Empirical correlation for determining the fraction of the liquid deposited on the walls of the tube can be casted in terms of the bubble drift velocity, $m$, given by:

$$
m=\frac{U_{b}-U_{s}}{U_{b}}
$$

Thus, the bubble velocity is somewhat faster than the average liquid slug velocity due to the presence of the liquid film around it. As this drift or relative velocity depends on the film thickness surrounding the bubble, it is obvious that the scaling for film thickness with Ca should, in general, be also applicable for $\mathrm{m}$. Indeed, as per [7], $\mathrm{m}$ scales as $\mathrm{Ca}^{1 / 2}$ while as per the [6] theory it scales as $\sim \mathrm{Ca}^{2 / 3}$. Such scaling typically applies to low $10^{-3}<\mathrm{Ca}<10^{-2}$. When inertia forces become dominant and the liquid film thickness gets limited by the thickness of the boundary layer that develops in the liquid as it moves, the parameter $\mathrm{m}$ asymptotically approaches a constant value $\sim 0.56-0.60$ at large Capillary numbers $[8,9,10]$. The presence of the film that separates the bubble from the wall means that the bubble velocity is not equal to the liquid one [11]. The presence of bubbles in front and at the back of the slugs, modifies the flow field in the liquid slug compared with single-phase flow and toroidal vortices extending the length of the slug can form [12, 13]. The recirculation patterns within the liquid slugs enhances heat and mass transfer from liquid to wall and interfacial mass transfer from gas/vapor to liquid. The transport mechanisms are thus influenced by the dynamics of isolated 'unit-cell', consisting of a Taylor bubble and the adjoining liquid plug. Understanding transport mechanism necessitates localized experimental observations of slug-bubble systems, with synchronized measurements of the resulting fluctuations in local conditions such as temperature, pressure and wall heat flux [14]. Such information is vital for designing microthermofluidic and micro-chemical reactor systems that operate in this regime. Bao et al., [15] experimentally investigated non phase-change air-water flow in a circular channel of diameter $1.95 \mathrm{~mm}$ under constant heat flux boundary condition and observed enhancement in heat transfer coefficient due to the presence of gas bubbles. Narayanan and Lakehal, [16] conducted numerical simulation of sensible heat transfer during gas-liquid flow in small-diameter pipes and found that overall heat removal is significantly increased due to the presence of circulation as compared to the corresponding 
single-phase flow. Walsh et al. [17] experimentally investigated the heat transfer rate in non-boiling two phase flow problem in a circular channel of $1.5 \mathrm{~mm}$ of internal diameter under constant heat flux boundary conditions. They used infra red thermography to record the change in substrate temperature, to calculate the Nu. They showed that such flow pattern can be very useful for augmentation of $\mathrm{Nu}$ in fully developed flows, whereas, in contrast the performance degrades in case of entrance region flow. However, these observations were made without measurement of the local fluid temperature; the fluid temperature was extrapolated.

In this work, we undertake local heat transfer measurements of isolated air-water Taylor bubble/ Taylor bubbletrain flow, in a square channel (aspect ratio $\approx 1$ ). A square channel is chosen as it provides the least wetted perimeter, for a given cross sectional area and therefore is preferable from an overall pressure drop point of view. Flow Reynolds number, $\operatorname{Re}$ (based on liquid superficial velocity, $\mathrm{J}_{1}$ ) and the volumetric flow rate of air are the variable parameters. As the channel is cut at the one face of the solid substrate and the constant heat flux is applied to the opposite face. So this problem inherently involves the heat conduction in the substrate to play an important role in the analysis of the thermal behaviour, [18]. From this view point, benchmarking of the setup has been done by performing some fundamental experiments and results are validated against the available theoretical observations, before performing the actual tests.

\section{Experimental Details and Procedure}

The primary idea of the experimental design is to create an extended surface fin structure, one end of which is subjected to a constant heat flux boundary condition and the other end is subjected to a convective heat transfer boundary condition. As the heater is located away from the channel, the thermal boundary condition at the fluid-wall interface is not identified as the conventional Uniform Heat Flux (UHF) or Uniform Wall Temperature (UWT). Such a configuration, which is frequently encountered in real-time applications, makes the problem inherently conjugate in nature. The goal is to reduce the effect of axial conduction through the solid fin substrate in the streamwise direction and simultaneously to reduce the effective Biot number in the transverse direction at the channel wall, so as to get a good estimate of the local heat transfer coefficient. To establish this methodology for correct estimation, the conjugate nature of the problems needs to be fully understood and the design of the setup should be such that effects of axial conduction on the estimation of local heat transfer are quantifiable and as far as possible, minimized. So, to observe and to reduce the effect of axial conduction, two different test sections are fabricated by using stainless steel $(\mathrm{k}=16 \mathrm{~W} / \mathrm{m}-\mathrm{K})$ and aluminum $(k=180 \mathrm{~W} / \mathrm{m}-\mathrm{K})$ with different geometrical configurations based on criterion derived in [18, 19]. The experimental facility, to meet the above requirements, is schematically shown in figure 1 . The test section consisted of single square mini-channel $\left(D_{h}=5.0 \mathrm{~mm}\right)$, machined on a substrate. The top of the channel was covered by a polycarbonate sheet $(5.0 \mathrm{~mm}$ thick, $\mathrm{k}=0.22 \mathrm{~W} / \mathrm{mK})$, providing necessary insulation from top of the test section, as shown in the figure 1. An unheated duct length of $200 \mathrm{~mm}$, having the same flow cross-section, was provided before the entry to the heated test section for achieving hydrodynamic flow development under operating flow Reynolds numbers covered in this work. The dimensional details of the test sections are provided in table 1.

Table 1. Dimensional details of test sections

\begin{tabular}{|l|c|c|}
\hline Parameters & Stainless Steel & Aluminum \\
\hline Heated Length, $\mathrm{L}$ & $300 \mathrm{~mm}$ & $140 \mathrm{~mm}$ \\
\hline Width, W & $25 \mathrm{~mm}$ & $45 \mathrm{~mm}$ \\
\hline Thickness, $\delta$ & $15 \mathrm{~mm}$ & $15 \mathrm{~mm}$ \\
\hline Hydraullic Diameter, $\mathrm{D}_{\mathrm{h}}$ & $5 \mathrm{~mm}$ & $5 \mathrm{~mm}$ \\
\hline Area of cross section, $\mathrm{A}$ & $5 \mathrm{~mm} \times 5 \mathrm{~mm}$ & $5 \mathrm{~mm} \times 5 \mathrm{~mm}$ \\
\hline
\end{tabular}

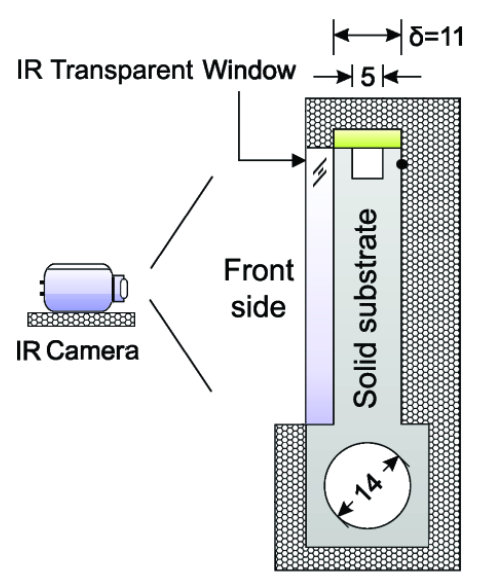

Thermal Insulation

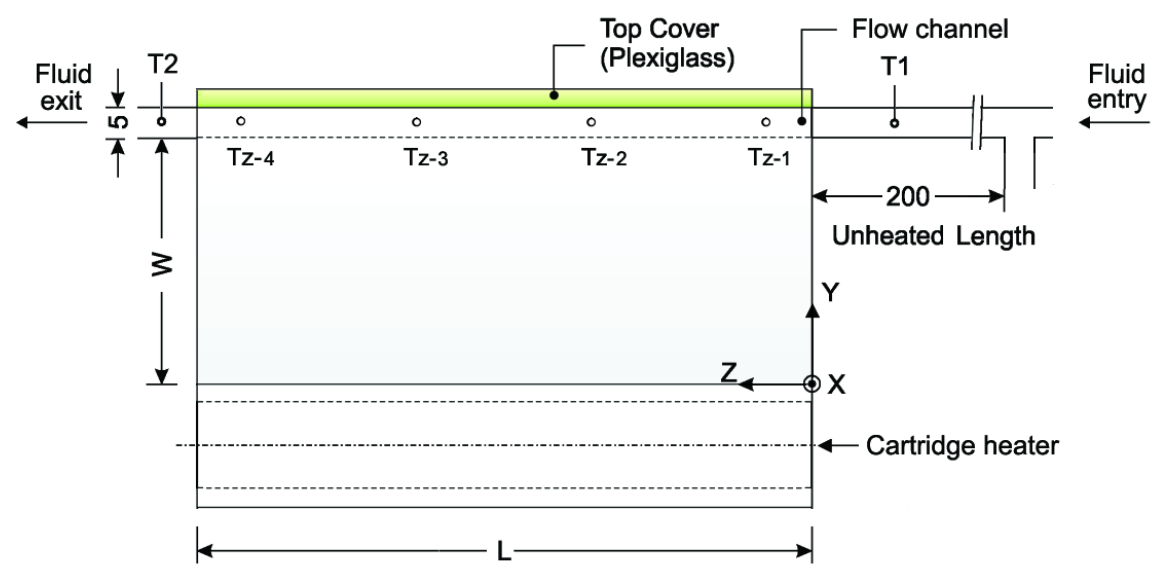

Fig. 1. Schematic of experimental setup 
For providing constant heat flux boundary condition at the base of the substrate, a cylindrical DC supplied cartridge heater (diameter: $15 \mathrm{~mm}$ ) was inserted, with the application of conducting thermal paste (metal oxide filled silicon oil paste supplied by RS components ${ }^{\circledR}, k=2.9 \mathrm{~W} / \mathrm{mK}$ ), as shown; as has been noted earlier, the upper surface of the flow channel was insulated. Digital multi-meters (accuracy $\pm 0.1 \mathrm{~V}$ and $\pm 0.01 \mathrm{~A}$ ) were used to measure the electrical power dissipation of the heater. Degassed and deionized water as liquid phase and dry air as gaseous phase are used as working fluids for the present experiments. Water at a fixed temperature, maintained by a constant temperature bath (Make: Julabo® F34 ME, accuracy $\pm 0.1 \mathrm{~K}$ ), was made to pass through the mini-channel and metered amount of air is supplied from pressurized cylinder through T-junction. The fluid temperatures at inlet and outlet and 3 other locations in the heated zone of the test section were measured by suitably located K-type micro thermocouples $($ Omega $(0.5 \mathrm{~mm}$ bead diameter, accuracy \pm 0.1 after calibration). All the surfaces of the substrate were completely insulated except an area of $25 \mathrm{~mm} \times 290 \mathrm{~mm}$ (in SS test section) at the front surface which was covered by a $5 \mathrm{~mm}$ thick an Infra-red transmitting CaF2 glass window (Make: Crystran®; Transmissivity: 95\%, for wavelength $2 \mu \mathrm{m}-7 \mu \mathrm{m}$; Refractive index = 1.399 at $5 \mu \mathrm{m} ; \mathrm{k}=9.71 \mathrm{Wm}-1 \mathrm{~K}-1$ ) to facilitate the recording of spatial IR thermographic profiles of the front surface of the substrate. For bench-marking the camera, and to measure the channel wall temperature at the back surface (behind the surface on which IR transparent glass was fitted; see figure 1), four more K-type pre-calibrated (against Pt-100 NIST traceable calibration curve) thermocouples $T_{z-1}$ to $T_{z-4}$ were attached in the flow direction along the channel, as shown in figure 1. Thermocouple data acquisition was carried out at $30 \mathrm{~Hz}$ and $50 \mathrm{~Hz}$ (depending on the volume fraction) by using a high precision 24 bit PCI-DAQ card (Make: National Instruments ${ }^{\circledR}$, NI 9213). The IR camera used (Make: FLIR, Model: SC4000; Indium Antimonide detector array) had an operational spectral band of 3-5 $\mu \mathrm{m}, 14$ bit signal digitization and a Noise Equivalent Temperature Difference of less than $0.02 \mathrm{~K}$ at $30^{\circ} \mathrm{C}$. IR images were acquired and post processed by using ThermaCAM ${ }^{\mathrm{TM}}$ Researcher-V2.9 software. Photron-Fastcam ${ }^{\circledR}$-SA3 high speed camera is used to visualize the slug flow in the unheated part of the setup. The images are acquired at $500 \mathrm{fps}$ for all experiments. Heat losses to the environment from the setup were estimated based on the conservation of energy, the difference between the electrical energy supplied to the channel and that received by the working fluid after steady state operation was achieved; this was found to be always less than $\sim 7-13 \%$, depending on the heat input. The Prandtl number of the fluid, as reported here, is based on the fluid temperature at the channel inlet.

\subsection{Experimental Data Reduction}

The peripheral average local Nusselt number $\left(\mathrm{Nu}_{z}\right)$ for the square duct used in the present study is defined as:

$$
\begin{aligned}
& \mathrm{Nu}_{\mathrm{z}}=\mathrm{h}_{\mathrm{z}} \cdot \mathrm{D}_{\text {hyd }} / \mathrm{k}_{\mathrm{f}} \\
& \mathrm{h}_{\mathrm{z}}=\mathrm{q}_{\mathrm{z}}^{\prime \prime} /\left(\mathrm{T}_{\mathrm{wm}}-\mathrm{T}_{\mathrm{fm}}\right) \\
& \mathrm{q}_{\mathrm{z}}^{\prime \prime}=-\mathrm{k}_{\mathrm{s}}(\partial \mathrm{T} / \partial \mathrm{y})_{\mathrm{z}}
\end{aligned}
$$

The heat flux ratio $\phi$ is defined as the ratio of the local heat flux $q_{z}^{\prime \prime}$ to that of the applied constant heat flux at the bottom of the substrate $q^{\prime \prime}$, i.e., $\phi=q_{z}^{\prime \prime} / q^{\prime \prime}$. For steady flow, local heat flux $q_{z}^{\prime \prime}$ is calculated from experimentally obtained IR thermogram as shown in figure 2, [20].

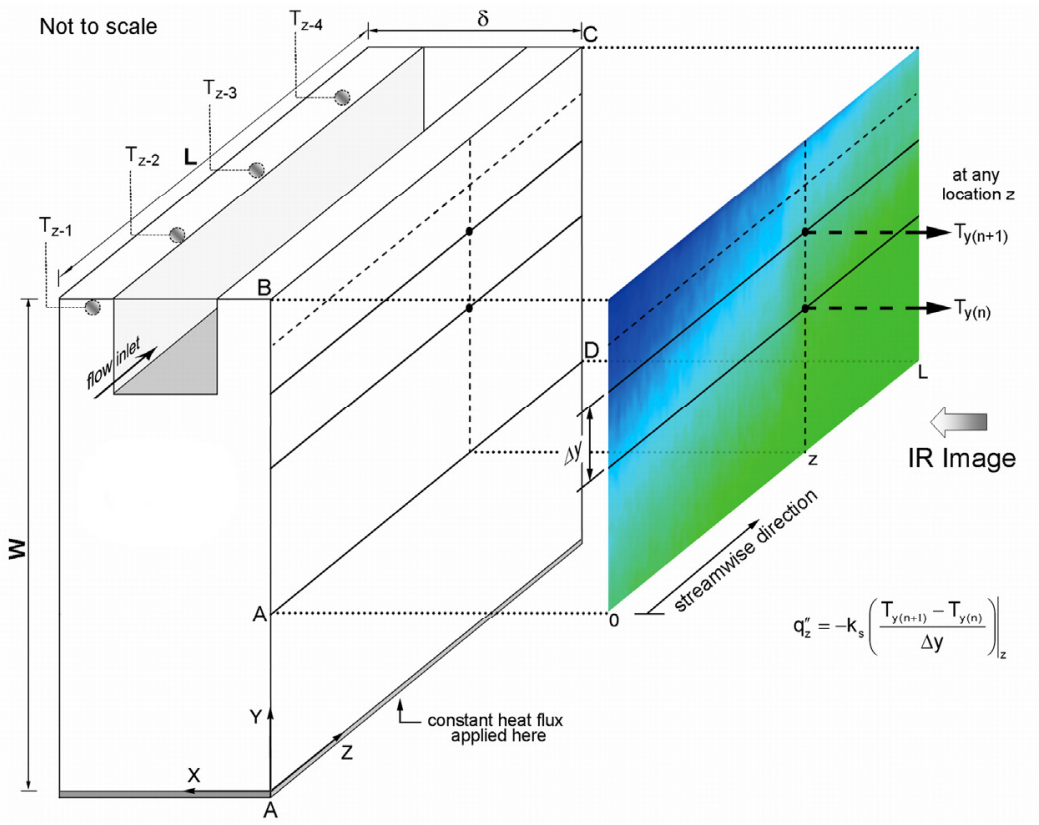

Fig. 2. Experimental data reduction scheme from the IR Thermogram 


\section{Results and Discussion}

Before discussing the results, it is very necessary to benchmark the experimental setup and the instrumentations to ascertain the correctness of the observations. Here, IR Camera is used for the surface temperature measurement. Its measurement depends on emissivity of the surface, distance of sensor from the object and room temperature and humidity. All other parameters except emissivity are known easily. To assess the emissivity in permissible uncertainty, surface is coated with high emissivity $(\varepsilon \geq 0.95)$ black paint. So, for getting correct measurement, assessment of emissivity of the surface is necessary. To assess the emissivity, the surface temperatures were measured by the thermocouples at several known locations and then tuning the value of emissivity such that the temperature reading matches with IR camera.

Now, to validate the experimental setup, we performed the single-phase steady state experiments on the both experimental setup (Al and SS) to investigate the heat transfer coefficient in the laminar hydrodynamically developed but in thermally developing flow at flow $\operatorname{Re} 300$. The results of the experiments are shown in figure 3 (a) and (b) and figure 4. Figure 3 (a), shows the wall and the bulk fluid temperature profile for $\mathrm{k}_{\mathrm{sf}}$ (ratio of thermal conductivity of the solid to the fluid) 300 and 26.6 and these profiles are validated with the theoretical temperature profile obtained for uniform heat flux (UHF). It is concluded that higher value of $k_{s f}$ increases the effect of axial conduction. The applied thermal condition is distorted significantly at the solid-liquid interface whereas for low $\mathrm{k}_{\mathrm{sf}}$ the effect of axial conduction is minimized. The temperature profile is in good agreement with the analytical solution. This is clearly observed in the IR thermogram, figure $3(b)$, that higher $k_{s f}$ tends to iso-thermalize the boundary condition at the solid-liquid interface. Figure 4 , shows the axial variation of the experimental Nusselt number and heat flux ratio for the $\mathrm{k}_{\mathrm{sf}}$ value of 26.6 and is compared with the numerical results. As it is clear from this figure that heat flux ratio $\phi$ is approximately axially constant i.e. at the solid liquid interface the thermal boundary condition of uniform heat flux (UHF). The heat flux ratio $\phi<1$ signifies that some amount of heat input is lost to the ambient. Thus, based upon these results, it is concluded that the stainless steel test section is good from the thermal boundary condition point of view. Therefore, all the slug-bubble train flow experiments were performed on the stainless steel substrate material.
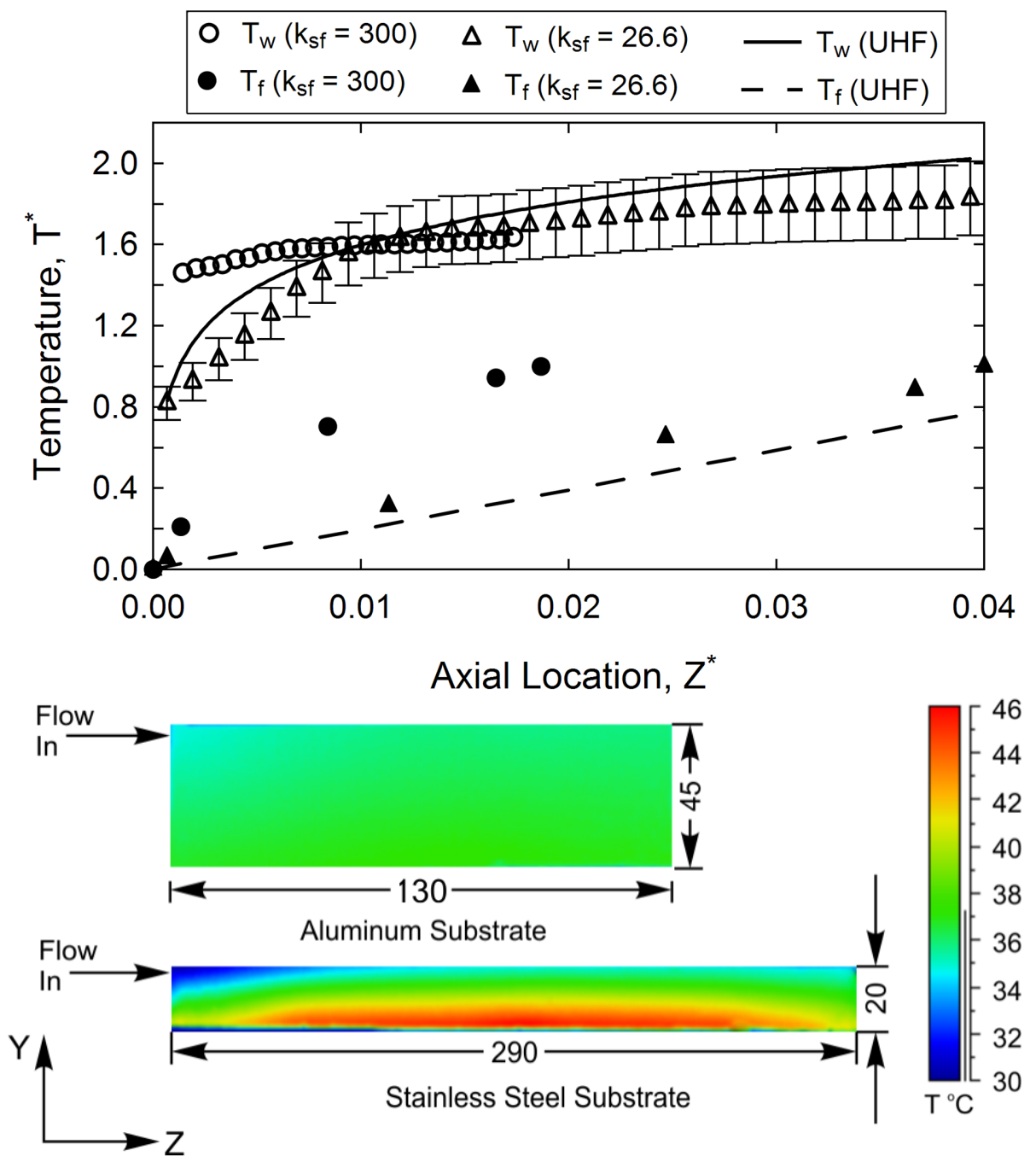

Fig. 3. (a) Axial variation Wall and bulk fluid temperature (b) IR Thermogram for $k_{s f} 300$ and 26.6 


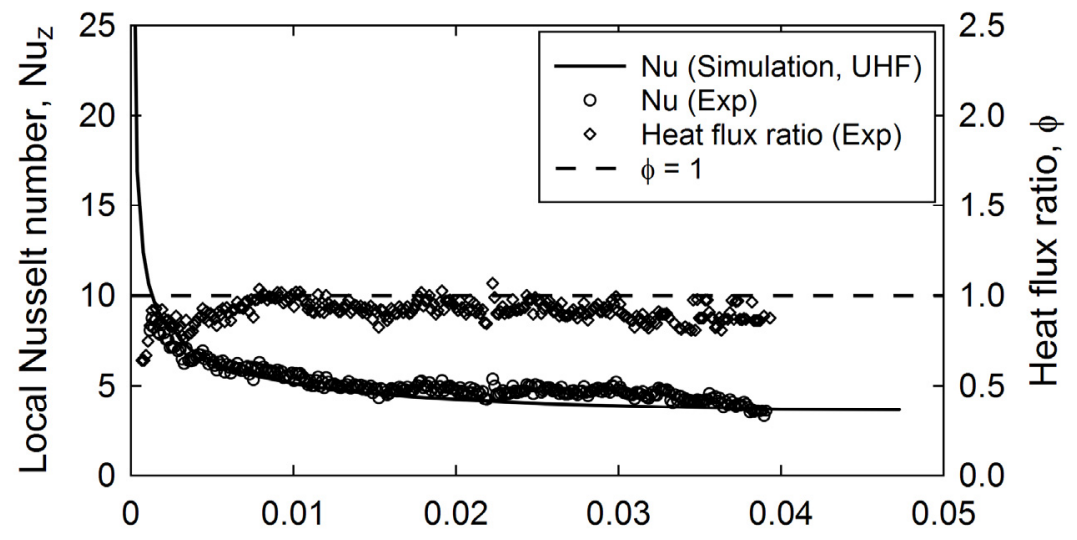

Axial Location, $Z^{*}$

Fig. 4. Axial variation of experimental Nusselt number, Nu and heat flux ratio

\subsection{Heat transfer analysis of slug flow}

The continuous introduction of a quasi-periodic Taylor bubble train into a Graetz type flow provides the opportunity to study its effect on the effectiveness of the heat removal capability. As noted earlier, Taylor bubble train flow has been achieved by injecting air from a pressurized cylinder, supplying air to the T-junction which is located far upstream. The flow rate of the air and therefore its volumetric flow ratio $(\beta)$ is controlled. Results reported here are from experiments carried out at different $\beta$ and with liquid flow $\operatorname{Re} 300$. The fully developed flow of the Graetz-solution suggests an asymptotic constant value of Nusselt number under the said boundary condition [21]. Hence, fully developed flows saturate the heat transfer coefficient. Thermal transport enhancement under such flow conditions can be achieved by creating local flow disturbances. In the present case, this is achieved by the injection of a quasi-periodic Taylor bubble train into the liquid flow. Figure 5(a) and (b) show the temporal variation of the fluid and wall temperature versus time at the measurement station 1,2 and 3 for a volumetric flow rate ratio $(\beta)$ of 0.714 and 0.384 respectively and figure 5 (c) shows the IR thermogram of wall temperature for steady state single phase flow and for steady state Taylor bubble train flow with decreasing volume fraction. It is clear from the thermogram that the surface temperature increases with decrease in volume flow ratio i.e., for $\beta=0.384$, the temperature of the substrate approaches to the singe phase steady state level. Nusselt number for Taylor bubble train flow is shown in figure 6 (d). it is observed that the Nu decreases with decrease in volume flow ratio $\beta$ for present experimental range and the enhancement in the heat transfer is approximately $2-2.5$ times of the liquid single-phase steady state value as also reported by [22].
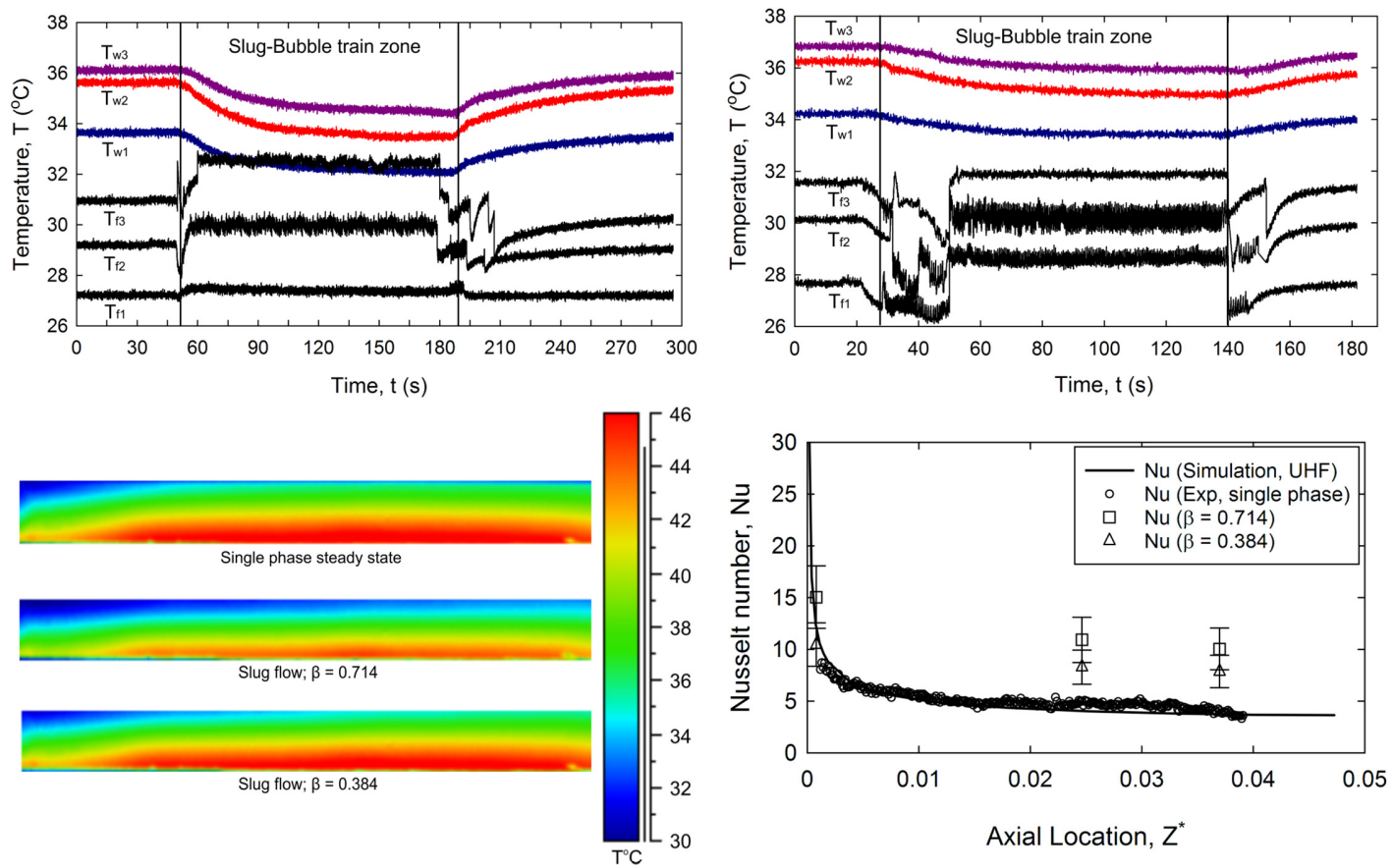

Fig. 5. Temporal variation of temperature (a) $\beta=0.714$ (b) $\beta=0.384$ (c) IR Thermogram of wall temperature for single phase steady state and slug flow for $\beta=0.714$ and 0.384 (d) axial variation Nu for Taylor bubble train flow 


\section{Summary and conclusions}

1. Injection of Taylor bubbles provides an efficient means of heat transfer enhancement, up to 2 to 2.5 times, as compared to fully developed laminar single-phase flow. The relative enhancement may not be attractive when compared to developing single-phase flows.

2. At any given location in the streamwise direction, injection of Taylor bubble train results in lowering of the wall temperature and increase of the average fluid temperature. IR Thermography is successfully employed to observe the effect of Taylor bubble train on the wall temperature field.

3. Increasing volumetric fraction $(\beta)$, for a fixed mass flow rate of the liquid, the T-junction flow mixing system resulted in longer air bubbles with more numbers, i.e. smaller liquid plugs entrapped between the bubbles. This increased the local $\mathrm{Nu}$, for the present range of experiments. However, such an increase may not be monotonous; this aspect needs further exploration.

\section{REFERENCES}

[1] Triplett K.A., Ghiaasiaan S.M., Khalik A., Le-Mouel S.I., McCord B.N., "Gas-Liquid Two-Phase Flow in Microchannels. Part I: Two-Phase Flow Pattern”, International Journal of Multiphase Flow, vol. 25, pp. 377-394, 1999.

[2] Ghiaasiaan S.M., Abdel-Khalik S.I., "Two-Phase Flow in Microchannels”, Advances in Heat Transfer, vol. 34, pp. 145-254, 2001.

[3] Taha T., Cui Z.F., "CFD Modeling of Slug Flow Inside Square Capillaries", Chemical Engineering Science, vol. 61, pp. 665-675, 2006.

[4] Angeli P., Gavriilidis A., "Hydrodynamics of Taylor Flow in Small Channels: A Review", Proceedings of IMechE, Part C; Journal of Mechanical Engineering Science, vol. 222, pp. 737-751, 2008.

[5] Wörner M., "A key parameter to characterize Taylor flow in narrow circular and rectangular channels", 7th Int. Conf. Multiphase Flow (ICMF 2010), Tampa, FL, (USA), 2010.

[6] Bretherton F. P., "The motion of long bubbles in tubes", J. Fluid Mech., vol. 10, pp. 166-188, 1961.

[7] Fairbrother F., Stubbs A. E., "The bubble tube method of measurement", J. Chem. Soc., vol. 1, pp. 527-529, 1935.

[8] Taylor G. I., "Deposition of a viscous fluid on the wall of a tube", J. Fluid Mech., vol. 10, pp. 161-163, 1961.

[9] Cox B.G., "An Experimental Investigation of Streamlines in Viscous Fluid Expelled from a Tube", Journal of Fluid Mechanics, vol. 20, pp. 193-200, 1964.

[10] Giavedoni M.D., Saita F.A., "The Axisymmetric and Plane Caseof a Gas Phase Steadily Displacing a Newtonian Liquid - A Simultaneous Solution to the Governing Equations", Physics of Fluids, vol. 9(8), pp. 2420-2428, 1997.

[11] Fabre J., Lińe A., "Modeling of Two-Phase Slug Flow", Annual Review of Fluid Mechanics, vol. 24, pp. 21-46, 1992.

[12] Thulasidas T. C., Abraham M. A., . Cerro R. L, "Bubble Train flow in capillaries of circular and square cross section”, Chemical Engineering Science, vol. 50, pp. 183-199, 1995.

[13] Thulasidas T.C., Abraham M.A., Cerro R.L., "Flow Patterns in Liquid Slugs during Bubble Train Flow inside Capillaries", Chemical Engineering Science, vol. 52(17), pp. 2947-2962, 1997.

[14] King C., Walsh E., Grimes R., "PIV Measurements of Flow within Plugs in a Microchannel", Microfluids and Nanofluids, vol. 3, pp. 463-472, 2007.

[15] Bao Z. Y., Fletcher D. F., Haynes B. S., "An experimental study of gas liquid flow in a narrow conduit", Int. Journal of Heat and Mass Transfer, vol. 43, pp. 2313-2324, 2000.

[16] Narayanan C., Lakehal D., "Two phase convective heat transfer in miniature pipes under normal and microgravity conditions", Journal of Heat Transfer, vol. 130,( 074502), 2008.

[17] Walsh A. P., Walsh J. E., Muzychka S. Y., "Heat transfer model for gas-liquid slug flows under constant flux", Int. Journal of Heat and Mass Transfer, vol. 53, pp. 3193-3201, 2010.

[18] Moharana M K., Agarwal G., Khandekar S., "Axial conduction in single-phase simultaneously developing flow in a rectangular mini-channel array", International Journal of Thermal Sciences, vol. 50, pp. 1001-1012, 2011.

[19] Maranzana G., Perry I., Maillet D., "Mini- and micro-channels: influence of axial conduction in the walls", Int. J. Heat Mass Transfer, vol. 47, pp. 3993-4004, 2004.

[20] Mehta B., Dhyani A., and Khandekar S., "Estimation of Laminar Single-phase Heat Transfer Coefficient in the Entrance Region of a Square Mini-channel using Infra-red Thermography", Proc. 21st National and 10th International ISHMT-ASME Heat and Mass Transfer Conference, Paper \#IND_02_041, Chennai, India, December 27-30, 2011.

[21] R. K. Shah and L. A. London, "Laminar flow forced convection in ducts", Academic Press inc, ISBN $0120200511,1978$.

[22] Gupta R. , Fletcher F. D., Haynes S. B., "CFD modelling of flow and heat transfer in the Taylor flow regime", Chemical Engineering Science, vol. 65, pp. 2094-2107, 2010. 